\title{
SYNTHESIS AND PROPERTIES OF
}

\section{POLYPYRROLE/POLYACRYLAMIDE COMPOSITE HYDROGELS}

\author{
Sangeeta Yadav ${ }^{1}$, R.M.Mishra ${ }^{2}$, Irfan Ahmad Mir ${ }^{3}$
}

\begin{abstract}
This study is purposed with the preparation of polyacrylamide/Polypyrrole hydrogel composite, in which the hydrogel of crosslinked polyacrylamide is a matrix and Polypyrrole is an electro-conducting component. The effects of synthesis conditions on the structure of the composite systems, their ability to swell in water, and mechanical characteristics are studied. The proposed synthesis procedure allows production of a bulk electro-conducting phase of Polypyrrole in the hydrogel matrix. The effect of crosslink density of hydrogel on the character of Polypyrrole distribution in the matrix is studied. The above composites combine the electrical characteristics of Polypyrrole with high elasticity and the ability to repeatedly swell the cross-linked poly (acrylamide).
\end{abstract}

Keywords: polyacrylamide, Polypyrrole, matrix, hydrogels, swell.

\section{INTRODUCTION}

For the development of a new generation of materials, so called stimuli-responsive systems that change their characteristics in response to an external action or under varying external conditions are of special interest. In this context, the attention of researchers has been focused on polymer hydrogels, which feature a marked response to various stimuli. Hydrogels based on cross-linked polyelectrolytes with a high degree of swelling not only absorb water in amounts exceeding their mass hundreds to thousands of times but also retain water at a certain pressure. The volume of a hydrogel changes during variations in temperature, $\mathrm{pH}$, the chemical composition of the surrounding medium, and the voltage of the external electrical field; hence, they are widely used as diverse promoting agents. From the practical viewpoint, among all possible means of activation, the electrical field has special importance because it permits effective control over the intensity of an external stimulus and integration of material in a space saving manner into electromechanical systems. However, owing to the low inherent conductivity of a hydrogel polymer network, the volume response is retarded greatly. This problem can be solved through the development of composite systems composed of a hydrogel matrix and a conducting component distributed in its volume. As this component conducting polymers that have long attracted close attention from researchers because of the promising advantages of their practical application in components of electronic, sensing, and electromechanical systems are of utmost interest [1, 2].At the same time, the preparation of electroactive hydrogels containing conducting polymers, such as polypyrrole and polyaniline, is complicated by their total insolubility in water and the thermodynamic incompatibility between components. The hydrogel-conducting polymer systems described in [3,4] are swelling powders that are sensitive to variations in temperature, the chemical composition of the medium, or the applied electrical voltage. However, the main drawback of the proposed procedures is the inability to produce bulk samples. The emerging difficulties are due to "embrittlement" caused by conducting polymers on the related composite materials. The main drawback is the poor mechanical characteristics, which impose serious limitations on the conducting polymers' practical application in technology. The objectives of this study are to develop a procedure for preparing electroactive composites directly in the bulk state via the in situ polymerization of pyrrole within a hydrogel matrix based on poly (acrylamide) (PAAm), to characterize the structure of the prepared systems, and to study their electrical characteristics and ability to swell in aqueous media with different $\mathrm{pH}$. To solve this problem, we employed PAAm gels. Their advantages over other hydrogels are related to the fact that these materials are exceptionally sensitive to changes in $\mathrm{pH}$. Other advantages of PAAm are the relative simplicity of its preparation and the fact that monomers for the synthesis of PAAm are less toxic than such a monomer as acrylamide, which is widely used for the synthesis of hydrogels.

\section{EXPERIMENTAL}

\subsection{Objects and Test Methods}

1 Synthesis of PPy by chemical oxidation polymerization, DBSA (Dodecyl benzene sulphonic acid) will serve as acidic medium while $\mathrm{FeCl}_{3}$ will be used as dopant. A dark black powder will be produced.

2 Hydrogels of PAAm with Methylenebisacrylamide (MBA) as crosslinking agent will be synthesized. Ammonium peroxydisulfate (PSA) will be used as initiator. Before jellification calculated $\mathrm{x}$ amount of PPy powder will be added. Hence PAAm/PPy composite will be synthesized.

$\mathrm{X}$ was estimated gravimetrically according to the equation.

$\mathrm{X}=$

$$
m_{\text {composite }}-m_{P A A m}
$$

$$
m_{\text {composite }}
$$


The hydrogel matrices were characterized by the degree of swelling in water and in a solution of hydrochloric acid. The data on swelling in acid were used to calculate the effective degree of crosslinking through the Flory equation for the gel swollen to equilibrium. The degree of swelling, $\mathrm{Q}$, of the PAAm hydrogels and composites was estimated gravimetrically and calculated as

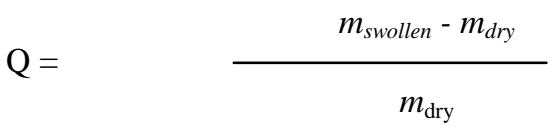

Where $m_{\text {dry }}$ is the weight of the sample in the dried state and $\mathrm{m}_{\text {swollen }}$ is the weight of the sample swollen to equilibrium.

\section{RESULTS AND DISCUSSION}

The morphological features of fractured surfaces of the composite hydrogel will be the typical scanning electron microscope (SEM) images of the PPy/PAAm composite hydrogel and characterization of the composite hydrogels will be undertaken by FTIR.

\section{REFERENCES}

[1] T. O. Ali, Surf. Coat. Technol. 200, 3918 (2006).

[2] M. Y. Guo, Y. Wei, A. G. MacDiarmid, and P. I. Lelkers, Biomaterials 27, 2705 (2006).

[3] G. Shi and G. Han, Thin Solid Films 515, 6986 (2007).

[4] Y. Wu, G. Alici, G. M. Sprinks, and G. G. Wallace, Synth. Met. 156, 1017 (2006)

[5] S. Machida, S. Miyata, and A. Techagumpuch, Synth. Met. 31, 311 (1989).

[6] Q. Tang, J. Lin, J. Wu, et al., Carbohydr. Res. 67, 332 (2007) 